\title{
¿Existe memoria larga en mercados bursátiles, o depende del modelo, periodo $o$ frecuencia?
}

\section{Is there Long Memory in Stock Markets, or Does it Depend on the Model, Period or Frequency?}

\author{
Héctor F. Salazar-Núñez* \\ Francisco Venegas-Martínez \\ Cuauhtémoc Calderón-Villareal
}

Recibido: 11 septiembre 2015/ Aceptado: 13 julio 2016

\section{Resumen}

El presente trabajo cuestiona si realmente existe memoria larga en los principales mercados accionarios del mundo $\mathrm{y}$, en caso de que esta exista, a qué se debe: ¿al tipo de modelos econométricos empleados, al periodo o la frecuencia de los datos? Para ello, se realiza un análisis comparativo entre modelos ARFIMA y GARCH. Los únicos mercados que mostraron resultados consistentes de memoria larga, independientemente del método, periodo y frecuencia, fueron China y Corea del Sur. El primero tiene memoria larga y el segundo, corta.

Clasificación JEL: N2, C58.

Palabras clave: Mercados bursátiles. Memoria larga. Métodos econométricos de series de tiempo.

\section{Abstract}

This paper analyses the existence of long memory in the major stock markets in the world, and if this is the case, whether it's due to the type of econometric models used, the period of study or the frequency of data

\footnotetext{
*Escuela Superior de Economía, Instituto Politécnico Nacional. Correo electrónico: hectorsalazar313@gmail.com

***Escuela Superior de Economía, Instituto Politécnico Nacional. Correo electrónico: fvenegas1111@yahoo.com.mx

**** Departamento de Estudios Económicos, Colegio de la Frontera Norte, A.C. Correo electrónico: ccalderon17@outlook.com
} 
(intraday, daily, weekly, etc.)? To do this, we perform a comparative analysis between the empirical results of ARFIMA and GARCH models. The stock markets that showed consistent results of long memory, regardless of the method, the period and the frequency were China and South Korea. The first one exhibits long memory, and the other a short one.

JEL classification: N2, C58

Keywords: Stock Markets. Long Memory. Time Series Econometric Models.

\section{Introducción}

Existen muchas investigaciones sobre el fenómeno de memoria en índices bursátiles, entre los cuales se encuentran el trabajo pionero de Hurst (1951), el cual dio origen a modelos econométricos con memoria (corta o larga), o bien como el propuesto por Granger y Joyeux (1980) y Hosking (1981), llamado modelo autorregresivo fraccionalmente integrado de medias móviles (ARFIMA o FARIMA), o el Baillie et al. (1996), llamado modelo autorregresivo generalizado de heterocedasticidad condicional fraccionalmente integrado (FIGARCH), y todas sus variantes. Por otro lado, la manera de probar la existencia de memoria en una serie de tiempo ha ido cambiando, ya sea por la eliminación o introducción de nuevos argumentos o por la forma en que se estima el coeficiente de Hurst, lo cual conlleva a enfoques paramétricos, no paramétricos o semiparamétricos. Las pruebas más comunes son las propuestas por Geweke y Porter-Hudak (GPH) (1983), Higuchi (1988) y Robinson (1995), y algunas otras de tipo heurístico como el método del correlograma y el método de la varianza agregada (Taqqu et al., 1995).

La importancia de probar la existencia de memoria, ya sea corta o larga, en una serie de tiempo, proviene de dos aspectos. Primero, hay que escoger el modelo adecuado que logre captar el fenómeno, ya que los modelos tradicionales basados en la hipótesis de mercado eficiente de Malkiel y Fama 1970 (caminata aleatoria), en donde el dato relevante es el inmediato anterior, no permite retener en la memoria eventos muy remotos. En contraste, los modelos que captan la memoria suponen que toda la serie es importante, dado que el último dato de la serie de tiempo también impacta al primero en diferente proporción. En segundo lugar, hay que generar mejores pronósticos para la toma de decisiones, sean de carácter económico o financiero.

Los resultados de los trabajos que tratan de probar la existencia de memoria en índices bursátiles han sido marginales; por ejemplo, Cheung y Lai (1995) analizan dieciocho índices de mercado con frecuencia mensual en el periodo 1970-1992, y encuentran memoria larga en Austria, España, Italia y Japón. 
Estos autores destacan que hay que distinguir entre memoria en los rendimientos y memoria en la volatilidad. Sadique y Silvapulle (2001) analizan siete índices de mercado con frecuencia semanal entre 1993 y 1998. Estos autores encuentran memoria larga en Corea del Sur, Malasia, Singapur y Nueva Zelanda. Ellos también introducen la diferencia de estimar el coeficiente de Hurst en el dominio temporal y en el de frecuencias. Henry Olan (2002) analiza el índice de mercado con frecuencia mensual, en 19821998, de nueve países, y encuentra memoria larga en Corea del Sur. Este autor encuentra que existe poca evidencia de memoria larga en el resto de los índices, y que se debe más bien a burbujas especulativas y costos de transacción. Hiremath y Kamaiah (2011) analizan veinte índices de mercado con frecuencia mensual entre 1990 y 2000 , y encuentran memoria larga en Brasil, Chile y Estados Unidos, haciendo diferencia entre mercados emergentes y consolidados. Otros trabajos que analizan índices y sus componentes son los de Anoruo y Gil-Alana (2011), quienes analizan once índices de mercado de países africanos con datos de frecuencia diaria, con intervalos de estudio distintos en 1993-2006, y encuentran memoria larga en Egipto, Marruecos, Nigeria y Túnez. Asimismo, Bhattacharya y Bhattacharya (2012) estudian diez índices de mercado con frecuencia diaria en 2005-2011, y encuentran que Brasil, Chile, China, Corea del Sur, Hungría, India, Malasia, México, Rusia y Taiwán tienen memoria larga. Por otro lado, algunos estudios analizan índices y hacen hincapié en mercados en vías de desarrollo, como se muestra en el trabajo de Barkoulas et al. (2000), donde se analiza el índice de Grecia en 1981-1990 con frecuencia mensual, hallando memoria larga en dicho índice. Tolvi (2003) estudia el índice de Helsinki Stock Exchange y veinte activos de empresas, con datos diarios de 19872001, y encuentra memoria larga en índices y en activos, destacando que es común la memoria larga en pequeños mercados. Granger y Hyung (2004) examinan el índice S\&P500 con frecuencia diaria en 1928-2002. El análisis lo realizan por sub-periodos y encuentran fuerte evidencia de memoria larga en el índice, en términos absolutos; también encuentran que cuando existe rompimiento estructural en el modelo de estimación, no se distingue qué tipo de memoria tiene la serie. Por su lado, Oh et al. (2006), analizan siete índices, dos de los cuales los examinan en frecuencia intradía (S\&P500 y KOSDAQ). Estos autores normalizan los rendimientos por medio de la varianza, y encuentran que Alemania, Corea del Sur, Estados Unidos, Hong Kong, Francia, Japón y Reino Unido presentan eficiencia del mercado y que todas las series tienen memoria larga, con excepción de los dos índices analizados intradía. También muestran que los mercados emergentes presentan más volatilidad que los maduros. De igual forma, Danilenko (2009) examina el índice OMX Baltic durante 2000-2008. Este índice contiene a los sectores comunicaciones, energía, consumo, salud, financiero y tecnología, hallando memoria larga en dicho índice. Mukherjee et al. (2011), estudia el índice Sensex, un aproximado del índice de la India, con frecuencia diaria de 
1997-2009 en términos de rendimientos y niveles. Estos autores no encuentran memoria larga. Saleem (2014) analiza el índice de mercado y la eficiencia del mercado financiero de Rusia, y otros mercados como el petróleo, energía, bienes de consumo y telecomunicaciones, con frecuencia diaria, en 2004-2013, en forma de rendimientos, encontrando en los índices una alta correlación y memoria larga.

Este trabajo examina el fenómeno de memoria, ya sea corta o larga, de veinte índices de mercados de países en vías de desarrollo y desarrollados, con frecuencias diaria, semanal y mensual, para analizar si afecta el hecho de que un índice presente o no algún tipo de memoria debido a su periodicidad. En la segunda sección, se presenta de manera breve el concepto de memoria y su modelado; en la tercera, se mencionan las pruebas que detectan memoria; en la cuarta, se realiza un análisis estadístico de los índices de mercado y se aplican las pruebas de detección de memoria. Por último, se presentan las conclusiones.

\section{Definición de memoria larga}

Existen diversas definiciones de procesos estacionarios con memoria larga, cada una referida a un aspecto de la serie de tiempo, como puede ser la autocorrelación, varianza y densidad espectral. Los primeros que definieron un proceso estacionario con memoria larga fueron Himpel y McLeod (1978), con base en el comportamiento de las autocovarianzas. Estos autores encontraron en su estudio que algunas series de tiempo tenían una caída lenta. ${ }^{1}$ En esta investigación, se retoma la definición de memoria larga de Beran (1994), la cual es como sigue: Si $y_{t}$ es un proceso estacionario, entonces existe un número real $\alpha(0,1)$ una constante $c>0$ que satisfacen:

$\lim _{k \rightarrow \infty} \frac{\rho(k)}{c k^{-\alpha}}=1$

donde $\rho(k)$ son las autocorrelaciones de la serie $y_{t}$. Otras definiciones alternativas se pueden encontrar en Beran (1994), Granger y Hyung (2004) y Palma (2007). Por ejemplo, $\operatorname{var}\left(\bar{y}_{t}\right) \approx c T^{-\alpha}$ cuando $T \rightarrow \infty$ y $f(\omega) \approx c \omega^{-\beta}$ cuando $\omega \rightarrow 0$, donde $f(\omega)$ es la densidad espectral y $c$ es una constante positiva. En cada caso, $\alpha, \beta$ satisfacen $0<\alpha<1$ y $0<\beta<1$. Como puede observarse, la primera definición se refiere a la varianza y la segunda a la densidad espectral.

\footnotetext{
${ }^{1}$ Se dice que $y_{t}$ es un proceso estacionario con memoria corta y larga, si la suma de la autocovarianzas (autocorrelación) es sumable (finita), es decir, $\sum_{k=-\infty}^{\infty}\left|\gamma_{k}\right|=\infty$, con $\gamma_{k}=\rho_{k} \sigma_{y}^{2}$
} 
Un modelo que puede capturar este tipo de fenómeno fue propuesto por Granger y Joyeux (1980) y Hosking (1981). Por su parte, Hosking estableció una extensión del modelo ARIMA bajo la metodología Box-Jenkins (1976), en el que se propone que el modelo tuviera dos comportamientos. El primero trata con un modelo $\operatorname{ARIMA}(0, d, 0)$ y el segundo con $p \neq 0$ y $q \neq 0$. El modelo ARFIMA o FARIMA $(p, d, q)$ está representado por la siguiente ecuación:

$\alpha(L)(1-L)^{d} y_{t}=\beta(L) \varepsilon_{t} \quad \varepsilon_{t} \sim N\left(0, \sigma_{s}^{2}\right)$,

donde $y_{t}$ es la variable de estudio, $\alpha(L)=1-\alpha_{1} L-\cdots-\alpha_{p} L^{p}, \quad \beta(L)=$ $1-\beta_{1} L-\cdots-\beta_{q} L^{q}$ y son los polinomios del operador rezago $L,(1-L)^{d}$ es el operador rezago fraccional ${ }^{2}$ y $\varepsilon_{t}$ es un proceso ruido blanco con media cero y varianza constante. El parámetro $d$ es un número real que puede tomar valores en el intervalo cerrado $[-0.5,0.5]$. Si $d<0.5$, entonces $y_{t}$ es estacionaria y todas las raíces de $A R(p)$ están fuera del círculo unitario. $\mathrm{Si}$ $d>-0.5$, entonces $y_{t}$ es invertible y todas las raíces $M A(q)$ están fuera del círculo unitario. $\mathrm{Si}-0.5<d<0.5^{3}$, entonces $y_{t}$ es estacionaria e invertible, de tal manera que la función de autocorrelación $\rho_{k}$ existe y es finita cuando $k \rightarrow \infty\left(\lim k^{1-2 d} \rho_{k}\right.$ existe cuando $\left.k \rightarrow \infty\right)$.

El método para estimar el orden de integración $(d)$ de la serie $y_{t}$ se obtiene fuera del método propuesto por Hosking (1981), ya que calcula por medio del rango reescalado $(R / s)$. Asimismo, Sowell (1992) obtiene el orden de integración $(d)$ dentro del proceso a través de la estimación exacta máxima verosímil en el dominio del tiempo, y supone que $y_{t}$ se distribuye normal con media y varianza constante $(y \sim N(\mu, \Omega))$, entonces, la función de verosimilitud está dada por:

$$
l\left(\theta\left(d, \alpha, \beta, \sigma_{\varepsilon}^{2}\right)\right)=-\frac{T}{2} \ln (2 \pi)-\frac{1}{2} \ln |\Omega|-\frac{1}{2}(y-\mu)^{\prime} \Omega^{-1}(y-\mu)
$$

\footnotetext{
${ }^{2}$ Véase al respecto el desarrollo de Hosking (1981) a través del teorema del binomio, o el de Hamilton (1994) por medio de la asociación de derivadas parciales con el operador de rezago fraccional. También es importante mencionar que el parámetro $d$ captura memoria larga o reversión a la media dependiendo del valor que tome el parámetro. Por otro lado, con este tipo de modelos se realiza un análisis de corto plazo, a diferencia de otros modelos que pueden hacer análisis de efectos de largo plazo y equilibrio, por ejemplo, modelos de valuación de activos financieros como el CAPM o Valor en Riesgo.

3 Aunque Bloomfield (1985) establece que el parámetro $d$ pueda tomar valores en un conjunto cerrado de $[-1.0,0.5]$, conservando las características del modelo, es decir, si $d>-1.0$, entonces $y_{t}$ es invertible y todas las raíces $M A(q)$ están fuera del circulo unitario.
} 
donde $\Omega$ es la matriz de varianzas-covarianzas de $y-\mu$ у $\mu=X \beta$, y es la media. Para obtener los parámetros del modelo se debe maximizar $l$ con respecto al vector $\theta\left(d, \alpha, \beta, \sigma_{\varepsilon}^{2}\right)$, como lo indican Sowell (1992), Beran (1994) y Doornik y Ooms (2003).

En el modelo clásico ARIMA $(p, d, q)$, el análisis de memoria larga se refiere al comportamiento de la correlación, varianza y densidad espectral. Mientras que en el modelo ARFIMA $(p, d, q)$, se presenta una caída exponencial de las correlaciones, y se observa también una caída lenta a la tasa $k^{-\alpha}$. La varianza tiene una caída igual a $T^{-\alpha}$ y la densidad espectral tiene un polo que tiende a cero, igual a la tasa $\omega^{-\beta}$.

\subsection{Memoria corta y larga de rendimientos y volatilidad, y relaciones de equilibrio de corto y largo plazo}

Cheung y Lai (1995) analizan diversos índices en mercados bursátiles internacionales, de frecuencia mensual, y destacan que es importante distinguir entre memoria corta o larga en los rendimientos, y memoria corta y larga en la volatilidad. Asimismo, de acuerdo con Hosking (1981) y Hamilton (1994), el parámetro $d$ en el modelo $\operatorname{ARFIMA}(p, d, q)$ o FARIMA $(p, d, q)$ captura memoria larga o reversión a la media, dependiendo del valor que tome dicho parámetro. Es también importante distinguir entre memoria larga y equilibrio de largo plazo. En este sentido, hay otros modelos que permiten realizar un análisis de equilibrio de largo plazo; por ejemplo, los modelos de valuación de activos financieros como el CAPM, bajo el supuesto de agentes competitivos e información simétrica. Por último, es importante mencionar que los modelos de series de tiempo de vectores autorregresivos (VAR) y la metodología de cointegración, son útiles para identificar relaciones de largo plazo.

El concepto de memoria y su importancia en las finanzas es ampliamente discutido en Peters (1994) y (1996) con la hipótesis del mercado fractal, como hipótesis que contiene a la del mercado eficiente, es decir, como una general. Por ejemplo, este autor realiza un resumen de los modelos de corte econométrico y financiero, en el que resalta la importancia de los mismos. Al respecto, los modelos de corte financiero que son más comúnmente aceptados son los desarrollados por Hu y Øksendal (2003), quienes plantean varios modelos de corte financiero con base en el movimiento browniano y sus variantes. 


\section{Pruebas de memoria larga}

Existen diversos métodos para detectar memoria larga en una serie de tiempo $y_{t}$, aunque el primer método fue propuesto por Hurst (1951). ${ }^{4}$ El coeficiente de Hurst se obtiene por medio de la estimación de la siguiente ecuación a través del método de máxima verosimilitud:

$\ln \frac{R_{T}}{S_{T}}=c+H \ln T$

donde $\left(R_{T} / s_{T}\right)$ es la notación utilizada para el estadístico de rango reescalado, $R_{T}$ es el rango ajustado, $s_{T}$ es la desviación estándar, $c$ es la constante de proporcionalidad, $T$ es el número de datos por intervalo y $H$ es el coeficiente de Hurst.

El coeficiente de Hurst puede tomar valores en $0 \leq H \leq 1$, de tal manera que se pueden presentar los siguientes casos. Si $0<H<0.5$, la serie de tiempo $y_{t}$ presenta memoria corta, es antipersistente y los valores medios en el largo plazo regresan a su condicion inicial. Si $0.5<H<1.0$, la serie de tiempo $y_{t}$ presenta memoria larga, es persistente y se espera que la media en el largo plazo nunca regrese a su condición inicial. Si $H=0.5$, entonces es una caminata aleatoria, donde los eventos presentes no influyen en el futuro.

El coeficiente de hurst $(H)$ se relaciona con el orden de integración $(d)$ de una serie $y_{t}$, por medio de la siguiente ecuación: $d=H-0.5$. Por otro lado, Beran (1994), Taqqu et al. (1995), y Palma (2007) realizan un resumen de los métodos más utilizados para detectar memoria larga, entre los cuales se pueden mencionar el correlograma, el gráfico de la varianza, la prueba de Geweke y Porter-Hudak (1983), la prueba de Higuchi (1988) y el método de estimación semi-paramétrica de Robinson (1995) o el estimador local de Whittle.

Con el método del correlograma, derivado de la definición de memoria larga, se puede examinar la autocorrelación y a la autocorrelación parcial. ${ }^{5}$ Aunque este método es gráfico, también se puede obtener un orden de integración de

\footnotetext{
${ }^{4}$ Para el desarrollo de estos modelos véase Peters (1994) y Beran (1994). El uso del coeficiente de Hurst en modelos de corte econométrico o financiero dio origen a los modelos fraccionalmente integrados como alternativa a los modelos tradicionales, véase al respecto Peters $(1994,1996)$ y Hu y Oksendal (2003).

${ }^{5}$ Es importante destacar que si en la autocorrelación se observa una caída lenta que tiende a cero, por ejemplo, una caída hiperbólica igual a $k^{2 H-2}$ (con $1 / 2<H<1$ ), y si además se obtiene un orden de integración $d \rightarrow 0$, es difícil saber si la serie tiene memoria larga o no, ya que la serie $y_{t}$ se confundiría con una serie estacionaria con un orden de integración cero, esto es, un modelo ARMA $(p, q)$.
} 
la serie de tiempo, por medio de la estimación del valor de $H$, a través del método de máxima verosimilitud con la siguiente ecuación, para obtener la pendiente $\alpha_{1}$ :

$\ln |\rho(k)|=\alpha_{0}+\alpha_{1} \ln k$,

la cual es utilizada para obtener el coeficiente de Hurst como $H=\left(\alpha_{1} / 2\right)+$ 1.

El método de la varianza agregada ${ }^{6}$ proviene de la definición de memoria larga en una serie y este se relaciona con el anterior método, ya que cuando se observa una caída lenta en la autocorrelación, se incrementa la varianza de la media de la muestra (Beran, 1994). Si $y_{t}$ es un proceso estacionario con memoria larga, entonces $\operatorname{var}\left(\bar{y}_{t}\right) \approx c T^{-\alpha}$, donde $c>0$ y $\alpha=2 H-2$. Para obtener el valor estimado de $H$, se estima la siguiente ecuación:

$\ln s^{2}=\alpha_{0}+\alpha_{1} \ln x$

donde $s^{2}$ es el valor de la varianza de cada corte y, $x$ es el número de cortes. La pendiente de la regresión, la cual es negativa, es $\alpha_{1}=2 H-2$.

Higuchi (1988) propuso un método para calcular la dimensión fractal $(d=2-H)$, que se describe como el índice de irregularidad de las serie de tiempo, pero supone que el total de observación se comporta de manera similar a una de sus partes. Considere

$$
L_{m}(k)=\frac{\left\{\sum_{i=1}^{t-m}|X(m+i k)-X(m+(i-1) k)| \frac{t-1}{\left(\frac{t-m}{k}\right) k}\right\}}{k}
$$

donde $X$ es la variable de estudio, $m, k$ y $t$ son números enteros. El primero representa el inicio de la serie; el segundo, el corte de la serie y, por ende, el avance progresivo de la serie, y por último, $t$ es el total de observaciones, de tal manera que satisfaga $m \leq k \leq t$.

Para obtener la dimensión fractal y, por ende, el coeficiente de Hurst, se realiza una estimación por medio del método de máxima verosimilitud de la ecuación (8).

\footnotetext{
${ }^{6}$ El desarrollo de la metodología para obtener el coeficiente de Hurst puede consultarse, por ejemplo, en Beran (1994), Taqqu et al. (1995), y Palma (2007).
} 
$\ln L_{m}(k)=\ln c-\alpha \ln (k)$

donde $L_{m}(k)$ representa el resultado obtenido en la expresión (7) y $k$ el número de bloques realizados.

La prueba de Geweke y Porter-Hudak (1983) o GPH, también se conoce como método del periodograma. Considere la ecuación:

$\ln I\left(\omega_{k}\right)=\beta_{0}+\beta_{1} \ln \left[2 \operatorname{sen} \frac{\omega_{k}}{2}\right]^{2}$

donde

$$
I(\omega)=\frac{1}{2 \pi T}\left|\sum_{k=1}^{T} X_{k} e^{i k \omega}\right|^{2}
$$

es el periodograma, $\omega_{k}=\frac{2 \pi k}{T}$, son las frecuencias con su respectiva transformación con $\omega_{k}=(-\pi, \pi], \beta_{0}$ es la parte autónoma y $\beta_{1}$ es la pendiente. La estimación de la ecuación (9) se realiza por el método de máxima verosimilitud, el cual proporciona el orden de integración $-d=\beta_{1} \mathrm{e}$ indirectamente el coeficiente de Hurst $\left(H=1-\beta_{1} / 2\right)$ de la serie $y_{t}{ }^{7}$

El estimador local de Whittle, propuesto por Robinson (1995), es un método semiparamétrico que se basa en el periodograma y que solo especifica la densidad espectral en su forma paramétrica cuando $\omega \rightarrow 0$. Es decir, se trata con frecuencias cercanas a cero.

$f(\omega)=G(H)|\omega|^{1-2 H}$ cuando $\omega \rightarrow 0$

La función discreta del estimador local de Whittle está dada por

$$
Q(G, H)=\frac{1}{m} \sum_{k=1}^{m}\left(\frac{I\left(\omega_{k}\right)}{G \omega_{k}^{1-2 H}}+\ln G \omega_{k}^{1-2 H}\right)
$$

donde $m$ es un número entero menor a $T / 2$ y satisface las siguientes condiciones: $T \rightarrow \infty, 1 / m+m / T \rightarrow 0 \mathrm{y}$

$$
I(\omega)=\frac{1}{2 \pi T}\left|\sum_{k=1}^{T} X_{k} e^{i k \omega}\right|^{2}
$$

\footnotetext{
${ }^{7}$ De acuerdo con Shimotsu y Phillips (2002), en la ecuación (9) se reemplaza $2 \operatorname{sen} \omega_{k} / 2$ por $2 \ln \omega_{k}$ para la estimación.
} 
es el periodograma. La frecuencia está dada por, $\omega_{k}=\frac{2 \pi k}{T}$ con $\omega_{k}=$ $(-\pi, \pi]$. En este caso, se remplaza $G$ por su estimador, el cual se define como

$G=\frac{1}{m} \sum_{k=1}^{m} \frac{I\left(\omega_{k}\right)}{\omega_{k}^{1-2 H}}$

y se obtiene finalmente,

$R(H)=\ln \frac{1}{m} \sum_{k=1}^{m} \frac{I\left(\omega_{k}\right)}{\omega_{k}^{1-2 H}}-(2 H-1) \frac{1}{m} \sum_{k=1}^{m} \ln \omega_{k}$

La ventaja que presentan las pruebas de Geweke y Porter-Hudak (1983) y Robinson (1995), es que adicionalmente se pueden hacer simulaciones con los valores que puede tomar el coeficiente de Hurst $(H)$, por medio de la variación del número de cortes representado por el parámetro $\alpha$ dentro de la regla $m=T^{\alpha}(\operatorname{con} \alpha=0.5)$.

\section{Análisis empírico}

El periodo de estudio considera de 1997.12 a $2014.2{ }^{8}$ Este trabajo analiza los principales índices del mercado accionario con diferente frecuencia: diaria, semanal y mensual. Los datos diarios solo coinciden en el inicio y el final, no así en cantidad, debido a días festivos o días donde hubo cierre de la bolsa por alta volatilidad. Los índices de mercado que se examinan, son: MERVAL-AR, ATX-AT, EURONEXT BEL-20-BE, IBOVESPA-BR, S\&P/TSX Composite-CA, SMI-CH, SSE Composite Index-CN, DAX-DE, CAC 40-FR, FTSE 100-GB, HANG SENG Index-HM, Composite Index-ID, Nikkei 225-JP, KOSPI COMPOSITE Index-KR, IPC-MX, FTSE Bursa Malaysia KLCI-MY, STRAITS TIMES Index-SG, TSEC WEIGHTED Index-TW, NASDAQ Composite-US1 y S\&P 500-US2.

La gráfica 1 muestra los datos con frecuencia diaria, semanal y mensual, los cuales se presentan a escala logarítmica base 2 , y en unidades de cientos. Se observan movimientos similares de todas las series y el rompimiento más importante se da en 2008, debido a la crisis de hipotecas en los Estados Unidos que contagia a todos los índices de manera instantánea. Aunque también se observan otros cambios como los de 1998 y 2001, los cuales coinciden con la crisis asiática y la crisis de la burbuja.com, pero no fueron tan fuertes y contagiosas como se observa en la de 2008. La debacle de 2008,

\footnotetext{
${ }^{8}$ Los datos fueron obtenidos de yahoo finance.
} 
sin duda, afectó los tipos de cambio y los rendimientos en los indicadores accionarios de muchas economías. Para incorporar el impacto de la crisis financiera de 2008 en los índices bursátiles bajo estudio, se requeriría analizar la influencia de muchas otras variables de tipo cualitativo y cuantitativo, por ejemplo: la regulación del mercado financiero en los Estados Unidos de América, los tipos de cambio, la tasa de interés de EUA, etc., lo que va más allá del alcance de esta investigación. Un análisis de la crisis económica de 2008, en el contexto de la presente investigación, se puede consultar en Mian y Sufi (2008) y Machinea (2009).

Los días que, en promedio, las bolsas de valores del mundo podrían cotizar en un año son 261; de los cuales, los índices de mercado de países asiáticos y latinos son los países que menos días cotizan en promedio, ya sea que se tengan más días festivos o de alta volatilidad. En 1998, los índices de Argentina, Brasil, Hong Kong, Japón y Corea del Sur estuvieron cerradas al menos 15 días. En 2001, el índice de Yakarta estuvo 44 días cerrado, y el resto de índices como mínimo no cotizaron 10 días, con excepción del índice de China (el cual cotiza los 261 días al año, en promedio, hasta 2006). Los índices más estables en el año son el Dow Jones, S\&P 500 y FTSE 100. En el periodo 2011- 2013 se observa que todos los índices de mercado empiezan a tener problemas similares a los observados en 2001, incluyendo China, que no cotizó 24 días en el año 2013.

El cuadro 1 muestra los estadísticos de los diferentes índices de mercado con frecuencia diaria, los cuales fueron transformados a rendimientos logarítmicos, mostrando en general que los rendimientos son nulos. Los cambios en los rendimientos provienen de la volatilidad y estos normalmente son negativos, vistos por el sesgo; es decir, que solo los índices de Bélgica, Brasil, Hong Kong, Malaysia y México presentaron en este periodo más cotizaciones positivas que negativas. Por otro lado, se rechazó una distribución normal de los rendimientos en todos los índices, y todos se concentraron alrededor de la media, con puntos extremos, de ahí que la campana sea de tipo leptocúrtica y sesgada. Esto lo comprueba la prueba de Jarque-Bera, la cual rechaza la hipótesis de normalidad en todos los casos. La prueba aumentada de Dickey-Fuller (1979) muestra que —en todos los casos- ningún índice de mercado tiene una raíz unitaria, lo cual es indicio de estacionariedad, pero no de memoria larga. En este caso, y de acuerdo con Lee y Schmidt (1996), la prueba KPSS puede mostrar indicios de este fenómeno, con una hipótesis nula de estacionariedad contra la de integración fraccional; para ello, se realizaron pruebas con constante, y constante y tendencia. 

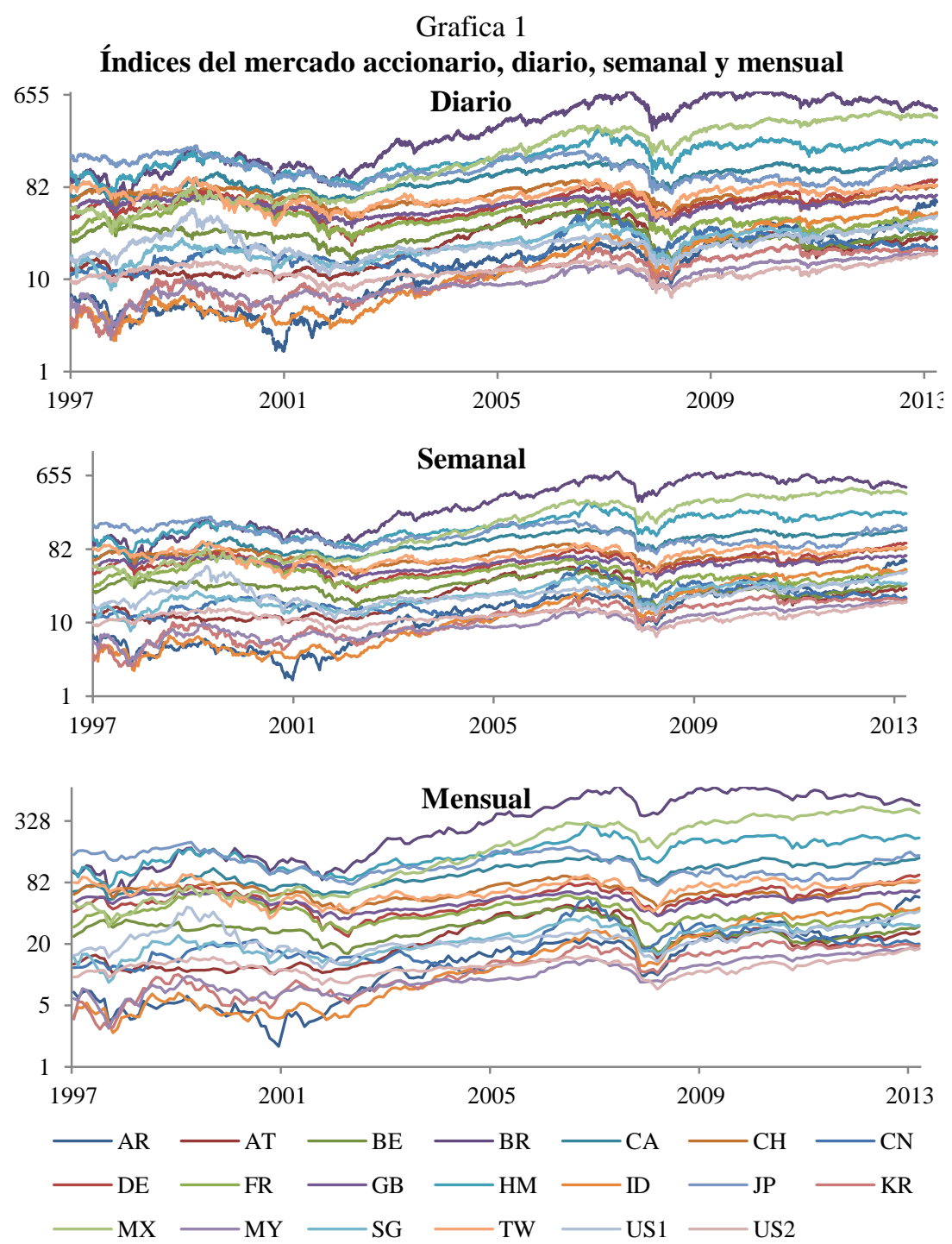

Nota: Elaboración propia con datos de finanzas yahoo.

Por otro lado, también el cuadro 1 muestra los estadísticos de los índices de mercado con frecuencia semanal, los cuales coinciden con la media a nivel diario, pero hay una disminución de la volatilidad en el sentido de que si sumamos la volatilidad diaria esta sería superior a la semanal; por eso, en general, solo hay un aumento alrededor de un $60 \%$. En cuanto al sesgo y la curtosis, se muestra que el primero solo aparece en los índices de Malaysia y 
China, los cuales generaron rendimientos positivos para el periodo de estudio con cotizaciones de frecuencia semanal, y estos fueron marginales debido a que la mayoría se concentró alrededor de la media y a que en pocas ocasiones las cotizaciones fueron extremas, lo cual da origen a una distribución de tipo leptocúrtica, y comprobada por la prueba de distribución normal que en todos los casos son rechazados. El resto de los índices de mercado tienen un sesgo negativo. La prueba Dickey-Fuller aumentada rechaza la hipótesis nula de raíz unitaria; en consecuencia, el polinomio de raíces tiene invertibilidad y, por lo tanto, se está en presencia de series estacionarias. Por último, la prueba de estacionariedad KPSS en ambas versiones acepta la hipótesis nula de estacionariedad. Por lo tanto, al igual que el cuadro anterior, no existe memoria larga en las series de tiempo.

Finalmente, en el cuadro 1, se observan los estadísticos de las series de tiempo con frecuencia mensual, en donde se observa que los rendimientos de Argentina, Brasil, Yakarta, Corea del Sur, México, Malaysia y el NASDAQ composite de Estados Unidos de América son diferentes de cero, en contraste con la periodicidad diaria y semanal. La volatilidad es estable si se compara con la diaria y semanal. El sesgo que presentan las series en su mayoría son negativas, con excepción de Corea del Sur y Malaysia, que tienen sesgo positivo, siendo este último índice el que en las tres frecuencia mostró un comportamiento positivo, lo cual quiere decir que, dentro del periodo de estudio, el índice de Malaysia en la mayoría de los casos tuvo rendimientos positivos por encima de la media. La distribución de los índices en su mayoría es del tipo leptocúrtico. Por lo tanto, la mayoría de los casos están cerca de la media con cotizaciones extremas, la excepción fue el índice de Francia y Taiwán, los cuales no rechazan normalidad. Esto se comprueba con la prueba de distribución normal Jarque-Bera, la cual dice que los rendimientos de Taiwán se distribuyeron normalmente, no así los de Francia. La prueba de estacionariedad KPSS acepta la hipótesis nula, por lo tanto, no todas las series muestran indicios de memoria larga.

En el cuadro 1, se muestra que la mayoría de los índices son volátiles y que su distribución no es normal, ya que estos se concentran alrededor de la media con algunos puntos extremos, dando origen a colas pesadas, este hecho se debió a diferentes crisis financieras, como por ejemplo las del 2001 y 2008, ya que en ambos casos se tienen picos pronunciados, como se observa en la gráfica 1. 


\section{Ensayos Revista de Economía}

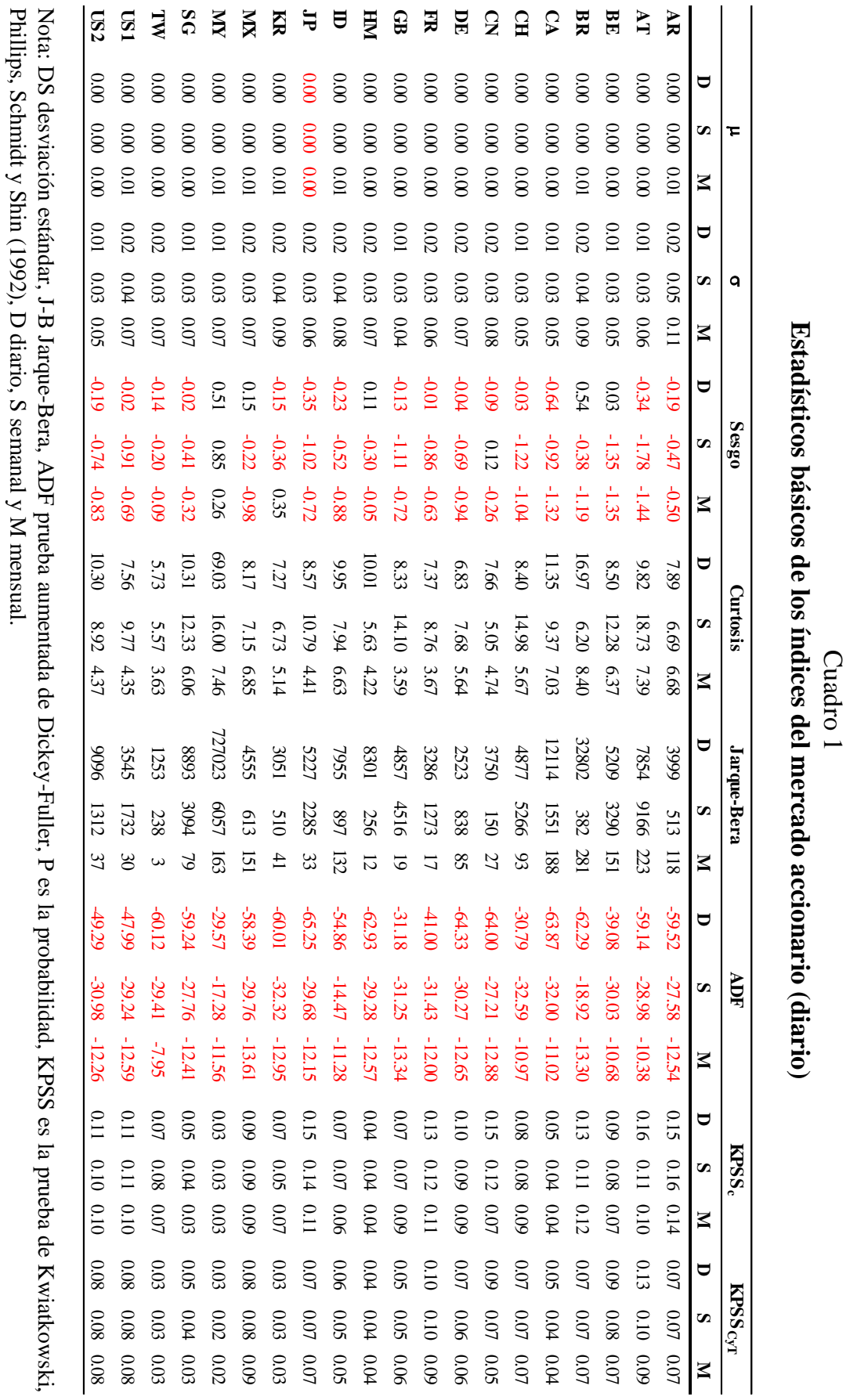


Cuadro 2

Estimación del orden de integración $d$

\begin{tabular}{|c|c|c|c|c|c|c|c|c|c|c|c|c|}
\hline & \multicolumn{3}{|c|}{$R / S$} & \multicolumn{3}{|c|}{ Correlograma } & \multicolumn{3}{|c|}{ Varianza } & \multicolumn{3}{|c|}{ Higuchi } \\
\hline & D & $\mathbf{S}$ & M & D & $\mathbf{S}$ & M & D & $\mathbf{S}$ & M & D & $\mathbf{S}$ & M \\
\hline $\mathbf{A R}$ & 0.15 & 0.18 & 9 & 4 & 9 & 6 & 4 & 0 & -0.01 & 0.00 & 1 & 1 \\
\hline AT & & 0.23 & 0 & 0.47 & 0 & 0 & 0.04 & 0 & 0.07 & -0.02 & 4 & 1 \\
\hline BE & 0 & 0.24 & 0 & 0.44 & 0 . & 0.40 & 1 & 0.08 & 4 & -0.04 & 0.02 & 01 \\
\hline BR & 0.07 & 0.17 & 0.0 & 0.48 & 0.46 & 0.23 & -0.01 & 0.00 & -0.08 & -0.05 & -0.01 & .06 \\
\hline $\mathrm{CA}$ & 0. & 0.24 & 0.19 & 0.45 & 0.40 & 0.19 & 0.01 & 0.03 & -0.14 & -0.04 & 0.02 & -0.01 \\
\hline $\mathbf{C H}$ & 0.06 & 0.26 & 0.11 & 0.42 & 0.17 & 0.44 & -0.03 & 0.04 & -0.05 & -0.06 & 0.01 & -0.05 \\
\hline $\mathrm{CN}$ & 0.04 & 0.24 & 0.09 & 0.40 & 0.23 & 0.05 & 0.06 & 0.15 & -0.15 & 0.00 & 0.05 & -0.13 \\
\hline DE & 0.01 & 0.20 & 0.16 & 0.42 & 0.28 & 0.03 & -0.01 & 0.03 & -0.08 & -0.05 & 0.01 & -0.09 \\
\hline FR & 0.10 & 0.22 & 0.18 & 0.43 & 0.18 & 0.34 & -0.02 & 0.07 & -0.08 & -0.08 & 0.02 & -0.05 \\
\hline GB & 0.08 & 0.22 & 0.12 & 0.42 & 0.25 & 0.36 & -0.06 & 0.00 & -0.10 & -0.09 & -0.02 & -0.07 \\
\hline HM & 0.20 & 0.16 & 0.17 & 0.44 & 0.37 & 0.17 & 0.00 & 0.01 & -0.20 & -0.04 & -0.02 & -0.13 \\
\hline ID & 0.13 & 0.13 & 0.15 & 0.47 & 0.44 & 0.20 & 0.04 & 0.05 & -0.08 & 0.01 & 0.04 & 0.05 \\
\hline $\mathbf{J P}$ & 0.11 & 0.08 & 0.18 & 0.43 & 0.31 & 0.38 & -0.01 & 0.03 & 0.02 & -0.08 & -0.03 & -0.03 \\
\hline KR & 0.11 & 0.20 & 0.15 & 0.47 & 0.43 & 0.25 & 0.01 & 0.01 & -0.30 & -0.04 & 0.00 & -0.04 \\
\hline MX & 0.12 & 0.18 & 0.15 & 0.48 & 0.45 & 0.25 & -0.01 & -0.03 & -0.05 & -0.02 & -0.01 & 0.13 \\
\hline MY & 0.18 & 0.23 & 0.22 & 0.46 & 0.41 & 0.22 & 0.05 & -0.02 & -0.43 & 0.03 & 0.02 & -0.12 \\
\hline SG & 0.26 & 0.21 & 0.22 & 0.44 & 0.35 & 0.18 & 0.05 & 0.02 & -0.25 & 0.00 & 0.03 & -0.10 \\
\hline TW & 0.26 & 0.18 & 0.16 & 0.36 & 0.17 & 0.06 & 0.03 & 0.00 & -0.12 & -0.04 & -0.02 & -0.21 \\
\hline US1 & 0.17 & 0.20 & 0.22 & 0.39 & 0.08 & 0.12 & 0.01 & 0.05 & -0.15 & -0.05 & 0.03 & -0.14 \\
\hline US2 & 0.14 & 0.16 & 0.12 & 0.40 & 0.12 & 0.31 & -0.03 & 0.02 & -0.10 & -0.08 & 0.00 & -0.11 \\
\hline
\end{tabular}

Nota: D-diario, S-semanal, M-mensual, d-orden de integración $(d=H-1 / 2)$.

En el cuadro 2, se muestra el orden de integración estimado por los diferentes métodos mencionados en la sección 3, con el método del correlograma y rango reescalado. Todos los índices de mercado tienen memoria larga, la cual varía según la periodicidad. El método del correlograma se distingue de las demás estimaciones, porque el orden de integración en la serie es mayor cuando se toma la frecuencia diaria, y menor cuando es mensual. En contraste con el método del rango reescalado, el orden de integración superior se presenta con datos semanales; las otras dos frecuencias son similares.

Con el método de la varianza, se observan varios hechos relevantes. Por ejemplo, con frecuencia mensual, las series se comportan con reversión a la media, es decir, ruido rosa; y con frecuencia diaria, los resultados son mixtos; en el caso semanal, es ruido negro en su mayoría. El método de Higuchi arroja resultados un tanto similares al anterior método, aunque - en este caso- las frecuencias mensual y diaria presentan comportamientos semejantes; el orden de integración en los índices sugeriría que —en el largo 
plazo - estos regresan a sus rendimientos medios. En el cuadro 2, también se observan varios resultados. Primero, el orden de integración de los índices de mercado cambia cuando varía la frecuencia. Segundo, esta variación depende del método que se utilice para su estimación; por ejemplo, la gráfica 2 muestra los cuatro métodos utilizados para la obtención del orden de integración, se observa que con el método de rango reescalado y del correlograma, todos los índices tienen memoria larga.

Con el método de la varianza y rango reescalado, los resultados son mixtos; es decir, con frecuencia mensual se obtiene un orden de integración negativo de los índices de mercado, lo cual indica memoria corta o de reversión a la media, significativa.

En el cuadro 3, se muestra la prueba de Geweke y Porter-Hudak para las frecuencias diaria, semanal y mensual. En primer lugar, la prueba se realizó utilizando diferentes cantidades de datos con $T^{0.45}, T^{0.50}$ y $T^{0.55}$, donde $T$ es el número de datos; para analizar si el orden de integración varía cuando se cambia la cantidad de datos utilizados en su obtención. El cuadro 5 muestra las estimaciones del orden de integración de cada uno de los índices de mercado, de los cuales solo los de Austria, Bélgica, Canadá, China, Corea del Sur, Indonesia, Malaysia, Singapur y Taiwán son estadísticamente diferentes de cero. De estos nueve índices de mercado, el orden de integración cambia de signo de positivo a negativo dentro de la misma frecuencia, lo cual es signo de volatilidad variante en los bloques que se utilizaron para la obtención del orden de integración. Por ejemplo, en Indonesia cambia de 0.07 a 0.16 , Corea del Sur de -0.09 a 0.08 , Malaysia de -0.08 a 0.07 y Singapur de -0.2 a 0.09 , con frecuencia diaria. Por otro lado, en los índices también se observa que el orden de integración, aunque no cambia de signo, varía demasiado. Por ejemplo, el índice de mercado de Canadá con frecuencia diaria cambia de 0.01 a 0.12 ; es decir, primero se comporta como una caminata aleatoria y después como una serie que posee memoria larga. Por último, el orden de integración varía de acuerdo con la periodicidad de que se trate; Taiwán con frecuencia diaria tiene indicios de memoria larga, y con mensual, como índice de memoria corta.

En el cuadro 4, se muestran los resultados obtenidos de aplicar la prueba del estimador local Whittle propuesto por Robinson (1995), los cuales son similares a los obtenidos con la prueba de Geweke y Porter-Hudak, ya que en los mismos índices de mercado, los cambios de signo de positivo a negativo y la variabilidad del orden de integración son parecidos. 
Gráfica 2

Orden de integración de acuerdo con el método utilizado
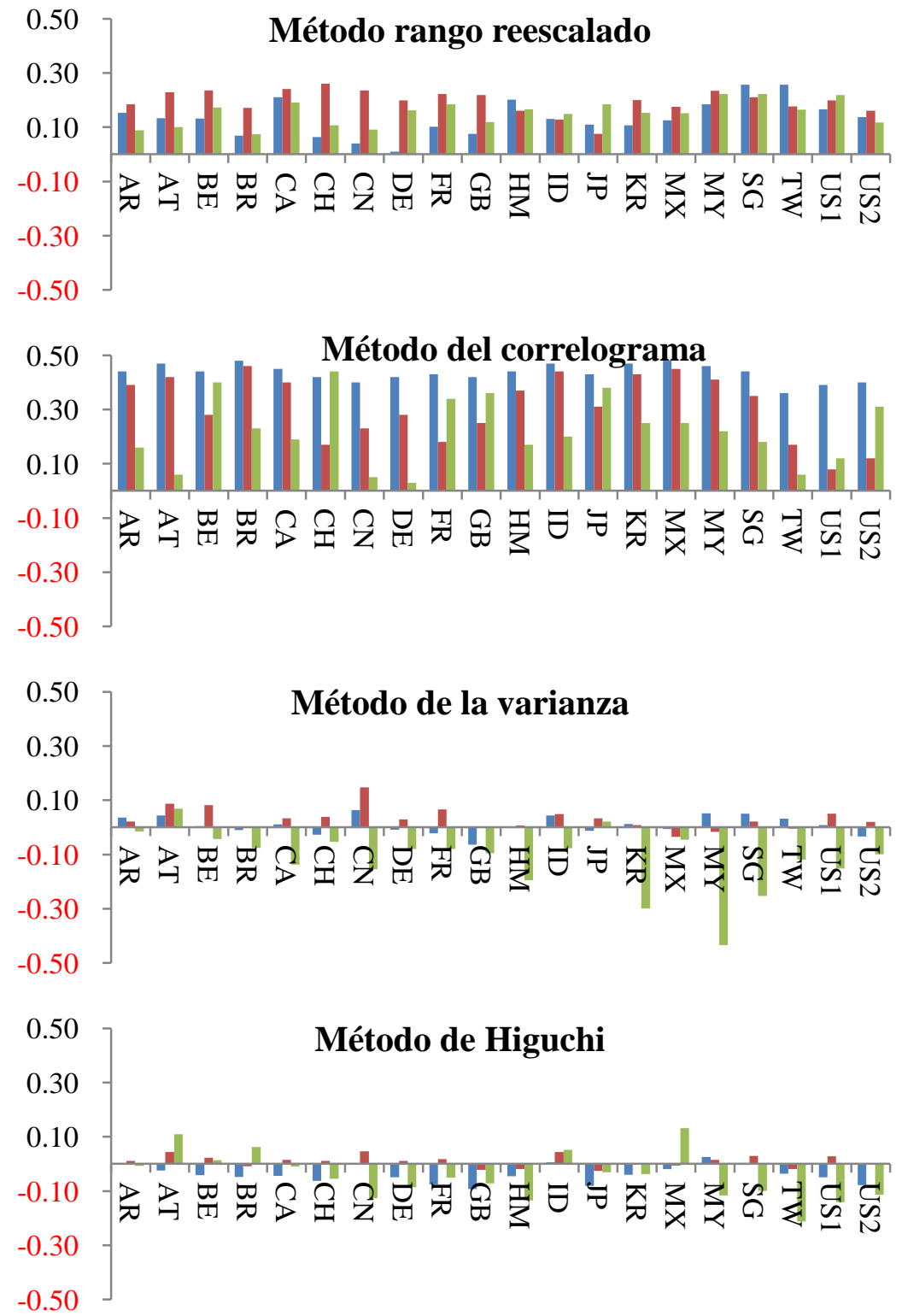

Nota: frecuencia, azul-diario, rojo-semanal y verde-mensual. 
Cuadro 3

Prueba de Geweke y Porter-Hudak, Diario, Semanal y Mensual

\begin{tabular}{|c|c|c|c|c|c|c|c|c|c|}
\hline & \multicolumn{3}{|c|}{$\mathbf{G P H}_{\mathbf{D}}$} & \multicolumn{3}{|c|}{$\mathbf{G P H}_{\mathbf{S}}$} & \multicolumn{3}{|c|}{$\mathbf{G P H}_{\mathbf{M}}$} \\
\hline & 0.45 & 0.5 & 0.55 & 0.45 & 0.5 & 0.55 & 0.45 & 0.5 & 0.55 \\
\hline$R$ & -0.04 & 0.06 & 0.03 & 0.03 & -0.02 & -0.02 & -0.11 & 0.00 & -0.12 \\
\hline $\mathbf{T}$ & 0.28 & 0.17 & 0.20 & 0. & 8 & 30 & 5 & -0.01 & 16 \\
\hline BE & 0.12 & 0.09 & 0.11 & 0.13 & 0.16 & 0.14 & -0.20 & -0.03 & 0.15 \\
\hline BR & 0.00 & -0.13 & 0.04 & -0.03 & 0.00 & -0.02 & 0.05 & 0.10 & 0.00 \\
\hline CA & 0.01 & 0.04 & 0.12 & -0.22 & -0.08 & 0.02 & -0.44 & -0.40 & -0.23 \\
\hline CH & 0.01 & 0.00 & 0.07 & -0.04 & 0.02 & 0.03 & -0.20 & -0.18 & -0.09 \\
\hline $\mathbf{C N}$ & 0.22 & 0.18 & 0.12 & 0.22 & 0.26 & 0.31 & -0.16 & 0.10 & 0.10 \\
\hline DE & -0.06 & 0.00 & 0.06 & -0.05 & -0.07 & -0.06 & -0.01 & 0.10 & -0.01 \\
\hline FR & 0.10 & 0.08 & 0.09 & -0.01 & 0.06 & 0.10 & 0.07 & 0.06 & 0.05 \\
\hline GB & 0.06 & 0.06 & 0.10 & 0.02 & 0.03 & 0.08 & -0.03 & -0.04 & 0.05 \\
\hline HM & -0.09 & -0.10 & 2 & -0.05 & -0.13 & 0.12 & -0.29 & -0.11 & -0.10 \\
\hline ID & -0.07 & -0.02 & 0.16 & 0.00 & 0.03 & -0.06 & -0.04 & -0.13 & -0.05 \\
\hline JP & 0.04 & -0.05 & -0.07 & -0.19 & -0.04 & 5 & -0.05 & -0.03 & -0.10 \\
\hline KR & -0.09 & -0.04 & & -0.24 & -0.15 & -0.10 & -0.63 & -0.36 & -0.35 \\
\hline MX & 0.03 & -0.02 & 0.04 & 0.00 & -0.03 & 0.03 & 0.26 & 0.09 & 0.03 \\
\hline MY & -0.08 & -0.04 & 0.07 & -0.29 & -0.38 & -0.14 & -0.68 & -0.54 & -0.48 \\
\hline SG & -0.02 & 0.04 & 0.09 & -0.08 & -0.14 & -0.01 & -0.32 & -0.26 & -0.18 \\
\hline TW & 0.04 & 0.07 & 0.03 & -0.14 & -0.04 & 0.09 & -0.41 & -0.22 & -0.23 \\
\hline US1 & 0.11 & 0.08 & 0.09 & 0.14 & 0.20 & 0.11 & 0.11 & 0.14 & 0.18 \\
\hline US2 & 0.16 & 0.04 & 0.10 & 0.12 & 0.12 & 0.14 & 0.09 & 0.03 & 0.06 \\
\hline
\end{tabular}

Nota: Elaboración propia con software Gretl, donde $\mathrm{GPH}_{\mathrm{D}}$ Diario, $\mathrm{GPH}_{\mathrm{S}}$ Semanal y $\mathrm{GPH}_{\mathrm{M}}$ Mensual.

Los resultados obtenidos en los cuadros 3 y 4 muestran que cada índice tiene un comportamiento diferente en cada periodicidad, aunque el intervalo de estudio no varía. Teniendo en cuenta este hecho, cada índice de mercado tiene que ser analizado y pronosticado. Por ejemplo, los inversionistas todos los días ven movimientos erráticos de varios índices de mercado, dado que no todos ellos invierten al mismo tiempo sino por etapas, los movimientos observados se desvanecen regresando a sus rendimientos medios mensuales. 
Cuadro 4

Estimador local de Whittle, Diario, Semanal y Mensual

\begin{tabular}{|c|c|c|c|c|c|c|c|c|c|}
\hline & \multicolumn{3}{|c|}{$\mathbf{E L W}_{\mathbf{D}}$} & \multicolumn{3}{|c|}{$\mathbf{E L W}_{\mathbf{S}}$} & \multicolumn{3}{|c|}{$\mathbf{E L W}_{\mathbf{M}}$} \\
\hline & 4.5 & 5 & 5.5 & 4.5 & 5 & 5.5 & 4.5 & 5 & 5.5 \\
\hline AR & -0.06 & -0.01 & 0.01 & -0.06 & -0.04 & -0.04 & -0.22 & -0.09 & -0.15 \\
\hline AT & 0.25 & 0.14 & 0.17 & -0.03 & 0.08 & 0.22 & 0.00 & -0.02 & -0.02 \\
\hline $\mathbf{B E}$ & 0.16 & 0.09 & 0.10 & 0.17 & 0.21 & 0.18 & 0.05 & 0.11 & 0.26 \\
\hline BR & 0.04 & -0.07 & 0.04 & -0.14 & -0.04 & 0.02 & -0.05 & -0.02 & -0.09 \\
\hline CA & 0.11 & 0.09 & 0.13 & -0.15 & -0.02 & 0.10 & -0.31 & -0.26 & -0.14 \\
\hline CH & 0.05 & 0.03 & 0.07 & 0.09 & 0.10 & 0.06 & 0.03 & 0.03 & 0.12 \\
\hline $\mathbf{C N}$ & 0.26 & 0.17 & 0.08 & 0.28 & 0.23 & 0.27 & -0.15 & 0.17 & 0.16 \\
\hline DE & 0.08 & 0.07 & 0.09 & 0.08 & 0.08 & 0.09 & 0.01 & 0.07 & 0.09 \\
\hline FR & 0.13 & 0.08 & 0.08 & 0.11 & 0.15 & 0.15 & 0.16 & 0.19 & 0.20 \\
\hline GB & 0.04 & -0.02 & 0.00 & 0.08 & 0.07 & 0.05 & 0.05 & 0.05 & 0.12 \\
\hline HM & -0.03 & -0.04 & 0.06 & -0.01 & -0.08 & -0.04 & -0.43 & -0.21 & -0.11 \\
\hline ID & -0.08 & -0.01 & 0.10 & -0.06 & -0.01 & -0.07 & -0.27 & -0.23 & -0.14 \\
\hline JP & 0.13 & 0.04 & 0.02 & 0.01 & 0.09 & 0.13 & 0.12 & 0.02 & 0.02 \\
\hline KR & -0.01 & 0.03 & 0.10 & -0.16 & -0.06 & -0.01 & -0.64 & -0.39 & -0.26 \\
\hline MX & 0.06 & -0.04 & 0.02 & 0.00 & -0.01 & 0.04 & 0.04 & -0.01 & 0.01 \\
\hline MY & -0.02 & 0.06 & 0.13 & -0.17 & -0.26 & -0.06 & -0.87 & -0.59 & -0.40 \\
\hline SG & 0.08 & 0.07 & 0.11 & 0.01 & -0.03 & 0.07 & -0.50 & -0.31 & -0.13 \\
\hline TW & 0.05 & 0.07 & 0.03 & -0.26 & -0.11 & 0.05 & -0.45 & -0.29 & -0.32 \\
\hline US1 & 0.06 & 0.05 & 0.08 & 0.10 & 0.09 & 0.06 & 0.09 & 0.15 & 0.18 \\
\hline US2 & 0.12 & 0.01 & 0.04 & 0.11 & 0.12 & 0.10 & 0.06 & 0.04 & 0.09 \\
\hline
\end{tabular}

Nota: Elaboración propia con software Gretl, donde $\mathrm{ELW}_{\mathrm{D}}$ Diario, ELW Semanal y ELW ${ }_{M}$ Mensual, 


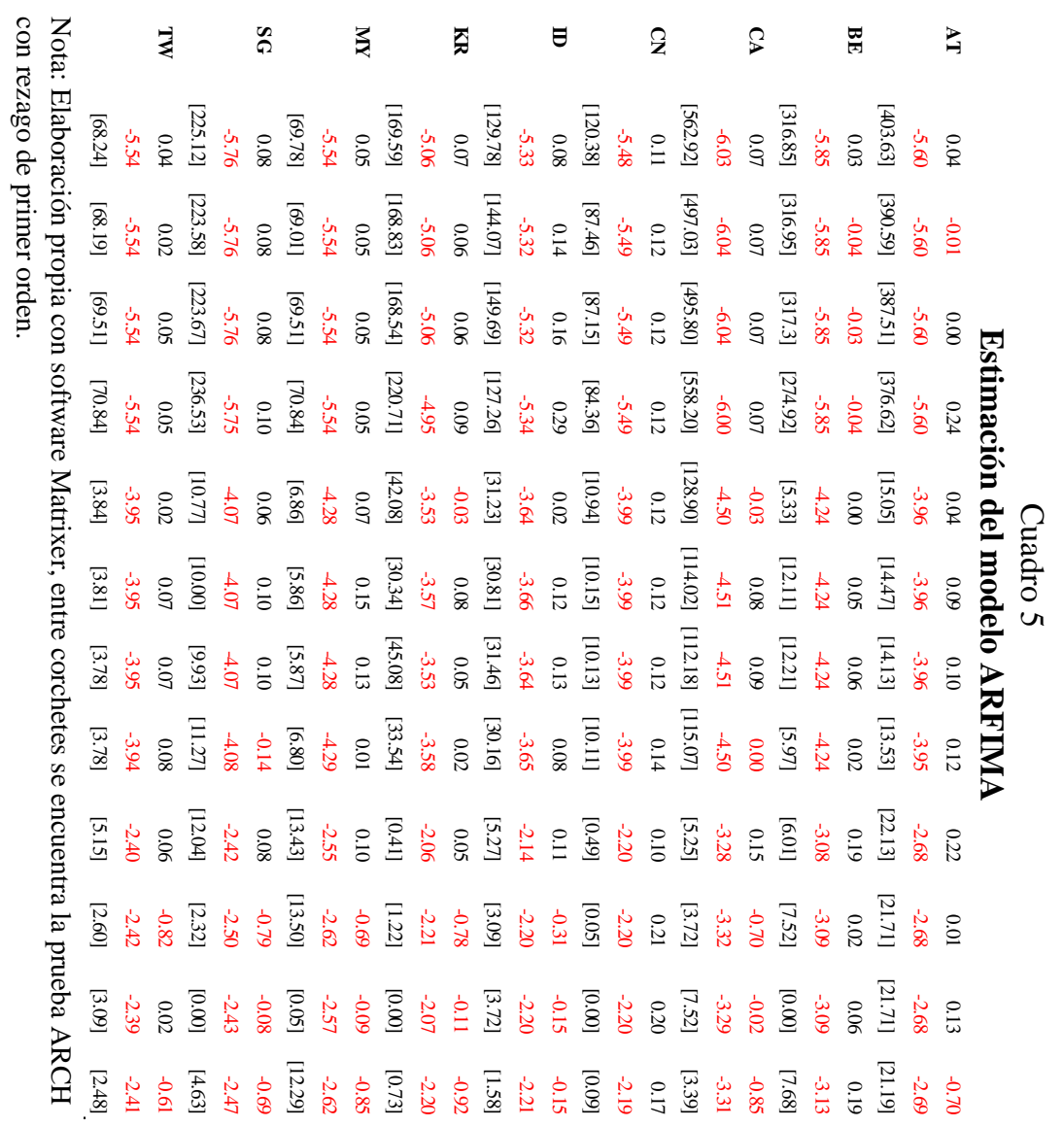


El cuadro 5 muestra los resultados de la estimación del modelo ARFIMA para cada uno de los índices de mercado que presentaron algún grado de memoria. Por medio del método propuesto por Sowell (1992), se realizó la estimación exacta con el método de máxima verosimilitud. Primero, con periodicidad diaria, se observa que todos los índices presentan memoria larga, aunque es marginal; solo China, Singapur y Yakarta presentan considerable memoria larga. Por otro lado, se muestra la prueba $\mathrm{ARCH}$, la cual tiene un valor alrededor de 2; por lo observado, la hipótesis nula debería ser rechazada. De esta manera, los resultados no son consistentes dado la relación no lineal de la varianza condicional y la variable independiente.

Por otro lado, el cuadro 5 muestra también, según se puede observar, las estimaciones para los índices de mercado con frecuencia semanal, que arrojan resultados similares, es decir, presentan memoria larga los índices de los países de Austria, China, Singapur y Yakarta; pero, también presentan indicios de $\mathrm{ARCH}$, al menos de primer orden, aunque esto disminuye considerablemente al transcurrir el tiempo. Finalmente, también en el cuadro 5 , se muestran los resultados obtenidos de la estimación del parámetro de integración $(\boldsymbol{d})$, junto con la prueba ARCH de primer orden, con periodicidad mensual, y de acuerdo con los resultados obtenidos, el índice de mercado de China es el único que presenta memoria larga sin problemas de $\mathrm{ARCH}$, al igual que Corea del sur, aunque en su caso es memoria corta. En los demás índices, se observa una clara influencia por los problemas de $\mathrm{ARCH}$, lo que genera variaciones que van de un memoria corta a larga, y viceversa. En este contexto, se esperaría que las cotizaciones del índice bursátil de China tuvieran cambios bruscos a la alza y a la baja, sin regreso al rendimiento medio y viceversa; mientras que para el índice bursátil de Corea del Sur, se esperaran cambios y, en un periodo largo, regresaría a su rendimiento medio.

Después de haber realizado el análisis estadístico y las pruebas de memoria, el índice de China y Corea del Sur, son los únicos que presentan el fenómeno de memoria. En el primer caso, se tiene memoria larga, y en el segundo, corta. El resto de los índices están influenciados por la volatilidad, típicas de series de corte financiero y, por lo tanto, la mejor forma de estimarlos es por medio el modelo GARCH, propuesto por Bollerslev (1986).

\section{Conclusiones}

En este trabajo, se aplicaron diferentes métodos para estimar el orden de integración de una serie de tiempo, partiendo del método seminal del rango reescalado y concluyendo con uno de los más recientes, el estimador local de Whittle, propuesto por Robinson (1995). Se observó que el orden de integración varía de acuerdo con el método que se utilice. Por ejemplo, con el 
método de rango reescalado y correlograma, todos los índices bursátiles tienen memoria larga, mientras que con el método de la varianza y Higuchi, los resultados son mixtos; es decir, pueden tener memoria corta con datos mensuales, y memoria larga con datos diarios, y viceversa. Con los métodos de Geweke y Porte-Hudak y el estimador local de Whittle, varios de los órdenes de integración de los diferentes índices de mercado no pasaron la prueba de significancia estadística, dejando solo a los índices de Austria, Bélgica, Canadá, China, Corea del Sur, Indonesia, Malaysia, Singapur y Taiwán, como candidatos a poseer algún tipo de memoria. Al analizar estos índices de mercado, se llegó a la conclusión de que varios de ellos eran sensibles a la información que los genera, observado un cambio de signo de positivo a negativo. Por otro lado, el orden de integración cambia cuando se modifica la periodicidad, sin modificar el intervalo de estudio, lo cual es indicio de que la estimación del orden de integración se ve contaminada por la volatilidad que presentan los índices diariamente, o porque los diferentes métodos de estimación del orden de integración no discriminan el fenómeno de la volatilidad. Por último, el índice de mercado de China presenta memoria larga con un orden de integración de 0.20 y un criterio de Akaike de -2.20, mientras que el índice de mercado de Corea del Sur es un proceso con reversión a la media, con un orden de integración de -0.11 y un criterio de Akaike de -2.07, ambos con frecuencia mensual. El principal mensaje de esta investigación es que debe tomarse en cuenta, para estudios futuros, que la periodicidad de una serie de tiempo cualquiera es relevante en el resultado, en virtud de los resultados mixtos que aquí se presentan.

Por último, es importante mencionar que en un proyecto futuro de investigación se realizará un análisis comparativo de diversas técnicas, con fundamentos financieros, distinguiendo sobre memoria larga y equilibrio de largo plazo; la tarea será monumental por el número de países estudiados; alrededor de 20.

\section{Referencias}

[1] Anoruo, E., and Gil-Alana, L. A. (2011). Mean Reversion and Long Memory in African Stock Market Prices. Journal of Economics and Finance, 35(3), 296-308.

[2] Baillie, R. T., Bollerslev, T., and Mikkelsen, H. O. (1996). Fractionally Integrated Generalized Autoregressive Conditional Heteroskedasticity. Journal of Econometrics, 74(1), 3-30.

[3] Barkoulas, J. T., Baum, C. F., and Travlos, N. (2000). Long Memory in the Greek Stock Market. Applied Financial Economics, 10(2), 177-184.

[4] Bhattacharya, S. N., and Bhattacharya, M. (2012). Long Memory in Stock Returns: A Study of Emerging Markets. Iranian Journal of Management Studies, 5(2). 67-88.

[5] Beran, J. (1994). Statistics for Long-Memory Processes. Vol. 61. CRC Press. 
[6] Bloomfield, P. (1985). On Series Representations for Linear Predictors. The Annals of Probability, 13(1), 226-233.

[7] Bollerslev, T. (1986). Generalized Autoregressive Conditional Heteroskedasticity. Journal of econometrics, 31(3), 307-327.

[8] Cheung, Y. W., and Lai, K. S. (1995). A Search for Long Memory in International Stock Market Returns. Journal of International Money and Finance, 14(4), 597-615.

[9] Danilenko, S. (2009). Long-Term Memory Effect in Stock Prices Analysis. Economics and Management, 14. 151-155

[10] Dickey, D. A., and Fuller, W. A. (1979). Distribution of the Estimators for Autoregressive Time Series with a Unit Root. Journal of the American statistical association, 74, 427-431.

[11] Granger, C. W., and Hyung, N. (2004). Occasional Structural Breaks and Long Memory with an Application to the S\&P 500 Absolute Stock Returns. Journal of empirical finance, 11(3), 399-421.

[12] Granger, C. W., and Joyeux, R. (1980). An Introduction to Long-Memory Time Series Models and Fractional Differencing. Journal of time series analysis, 1(1), 15-29.

[13] Geweke, J., and Porter-Hudak, S. (1983). The Estimation and Application of Long Memory Time Series Models. Journal of time series analysis, 4(4), 221238.

[14] Hamilton, J. D. (1994). Time Series Analysis, Vol. 2. Princeton: Princeton University Press.

[15] Henry, O. T. (2002). Long Memory in Stock Returns: Some International Evidence. Applied Financial Economics, 12(10), 725-729.

[16] Higuchi, T. (1988). Approach to an Irregular Time Series on the Basis of the Fractal Theory. Physica D: Nonlinear Phenomena, 31(2), 277-283.

[17] Himpel., K.W. and McLeod, A. I. (1978). Preservation of the Rescaled Adjusted Range. 2. Simulation Studies Using Box-Jenkins Models. Water Resour. Res. 14, 509-516

[18] Hiremath, G. S., and Kamaiah, B. (2011). Testing Long Memory in Stock Returns of Emerging Markets: Some Further Evidence. Economics, Management and Financial Markets, 6(3). 36-147.

[19] Hosking, J. R. (1981). Fractional Differencing. Biometrika, 68(1), 165-176.

[20] Hu, Y., and Øksendal, B. (2003). Fractional White Noise Calculus and Applications to Finance. Infinite Dimensional Analysis. Quantum Probability and Related Topics, 6(01), 1-32.

[21] Hurst, H. E. (1951). Long-Term Storage Capacity of Reservoirs. Trans. Amer. Soc. Civil Eng., 116, 770-808.

[22] Kwiatkowski, D., Phillips, P. C., Schmidt, P., and Shin, Y. (1992). Testing the Null Hypothesis of Stationarity against the Alternative of a Unit Root: How Sure Are We that Economic Time Series Have a Unit Root? Journal of econometrics, 54(1), 159-178.

[23] Lee, D., and Schmidt, P. (1996). On the Power of the KPSS Test of Stationarity against Fractionally-Integrated Alternatives. Journal of econometrics, 73(1), 285-302.

[24] Machinea, J. L. (2009). La crisis financiera internacional: su naturaleza y los desafíos de política económica. CEPAL, 33. 34-96 
[25] Malkiel, B. G., and Fama, E. F. (1970). Efficient Capital Markets: A Review of Theory and Empirical Work. The journal of Finance, 25(2), 383-417.

[26] Mian, A., and Sufi, A. (2008). The Consequences of Mortgage Credit Expansion: Evidence from the 2007 Mortgage Default Crisis. National Bureau of Economic Research, No. w13936.

[27] Mukherjee, I., Sen, C., and Sarkar, A. (2011). Long Memory in Stock Returns: Insights from the Indian Market. International Journal of Applied Economics and Finance, 5(1), 62-74.

[28] Oh, G., Um, C. J., and Kim, S. (2006). Statistical Properties of the Returns of Stock Prices of International Markets. arXiv preprint physics/0601126. http://128.84.21.199/pdf/physics/0601126.pdf

[29] Palma, W. (2007). Long-Memory Time Series: Theory and Methods, Vol. 662. John Wiley \& Sons.

[30] Peters, E. E. (1994). Fractal Market Analysis: Applying Chaos Theory to Investment and Economics. Vol. 24, John Wiley \& Sons.

[31] Peters, E. E. (1996). Chaos and Order in the Capital Markets: a New View of Cycles, Prices, and Market Volatility. Vol. 1. John Wiley \& Sons.

[32] Robinson, P. M. (1995). Gaussian Semiparametric Estimation of Long Range Dependence. The Annals of statistics, 23(5), 1630-1661.

[33] Sadique, S., and Silvapulle, P. (2001). Long-Term Memory in Stock Market Returns: International Evidence. International Journal of Finance and Economics,6(1), 59-67.

[34] Saleem, K. (2014). Modeling Long Memory In The Russian Stock Market: Evidence From Major Sectoral Indices. Journal of Applied Business Research (JABR), 30(2), 567-574.

[35] Shimotsu, K., and Phillips, P. C. (2002). Pooled log Periodogram Regression. Journal of Time Series Analysis, 23(1), 57-93.

[36] Sowell, F. B. (1992) Maximun Likelihood Estimation of Stationary Univariante Fractionally Integrated Time Series Models. Journal of Econometrics, 53, 165-188.

[37] Taqqu, M. S., Teverovsky, V., and Willinger, W. (1995). Estimators for LongRange Dependence: an Empirical Study. Fractals, 3(04), 785-798.

[38] Tolvi, J. (2003). Long memory in a Small Stock Market. Economics Bulletin,7(3), $1-13$. 